\title{
On the Existence of Weak Solutions for a Semilinear Singular Hyperbolic System
}

\author{
Joño-PAULO DIAS and MÁRIO FIGUEIRA*
}

ABSTRACT. In this paper we prove the existence of a weak solution for the semilinear singular real hyperbolic system

$$
\begin{cases}\frac{\partial u}{\partial t}+\frac{\partial u}{\partial r}+\frac{u-v}{r}+k\left(u^{2}+v^{2}\right) u=0 & \\ \frac{\partial v}{\partial t}-\frac{\partial v}{\partial r}+\frac{u-v}{r}+k\left(u^{2}+v^{2}\right) v=0 & , r \in \mathfrak{R}_{+}, t \in \mathfrak{R},\end{cases}
$$

where $k(r)$ is a smooth, bounded and positive function of the type $r^{r}, n \geq 3$, in a neighbourhood of zero. The initial data $\left(u_{0}, v_{0}\right)$ belong to $\left(\mathrm{H}^{2}\left(\mathfrak{R}_{\mathrm{r}}\right)\right)^{2}$ and verify

$$
u_{0}(0)=v_{0}(0), \frac{\partial u_{0}}{\partial r}(0)=-\frac{\partial v_{0}}{\partial r}(0),\left(r u_{0}, r \nu_{0}\right) \in\left(L^{2}\left(\mathfrak{R}_{+}\right)\right)^{2}
$$

\section{INTRODUCTION}

Let us consider the semilinear singular hyperbolic system

$$
\left\{\begin{array}{l}
\frac{\partial u}{\partial t}+\frac{\partial u}{\partial r}+\frac{u-v}{r}+k\left(u^{2}+v^{2}\right) u=0 \\
\frac{\partial v}{\partial t}-\frac{\partial v}{\partial r}+\frac{u-v}{r}+k\left(u^{2}+v^{2}\right) v=0
\end{array}\right.
$$

(*) Research supported by JNICT under contract n. 087568

1980 Mathematics Subject Classification (1985 revision): 35 L45.35 L60.

Editorial de la Universidad Complutense. Madrid, 1991. 
in the domain $\mathrm{D}=\left\{(r, t) \mid r \in \mathfrak{R}_{+}, t \in \mathfrak{R}_{\}}\right.$, where $(u, v): \mathrm{D}^{2} \rightarrow \mathfrak{R}^{2}, k: \overline{\mathfrak{R}}_{+} \rightarrow \overline{\mathfrak{R}}_{+}$, $k \in W^{1, \infty}\left(\mathfrak{R}_{+}\right)$and $k(r) \leq M r^{3}, k^{\prime}(r) \leq M r^{2}, r \in\left[0, r_{1}\right]$ for a certain $M>0$ and $r_{1}>0$.

The linear part of system (1.1) is associated (for complex $u$ and $v$ ) to a simplified model for the linear Dirac system (cf. [2] and [3]).

In order to study the Cauchy problem for the system (1.1) we regularise this system as follows:

$$
\left\{\begin{array}{l}
\frac{\partial u}{\partial t}+\frac{\partial u}{\partial r}+\frac{1}{r+\delta}(u-v)+k\left(u^{2}+v^{2}\right) u=0 \\
\frac{\partial v}{\partial t}-\frac{\partial v}{\partial r}+\frac{1}{r+\delta}(u-v)+k\left(u^{2}+v^{2}\right) v=0
\end{array}\right.
$$

where $0<\delta<1$. Given the initial data $\left(\begin{array}{l}u_{(0)} \\ y_{0}\end{array}\right)$, with a suitable smoothness. we first study the Cauchy problem for (1.2) under the boundary condition

$$
u(0, t)-v(0, t)=0, t \in \Re \text {. }
$$

Then we obtain some estimates on the solution, independent of $\delta$, and we pass to the limit, when $\delta \rightarrow 0$, in order to obtain a weak solution for the Cauchy problem for (1.1). More precisely, we prove the following theorem (where $\left.\mathrm{H}_{0, \ldots}^{1},(] 0, \mathrm{R}[)=\left\{u \in \mathrm{H}^{\mathrm{t}}(] 0, \mathrm{R}[) \mid u(0)=0\right\}\right)$ :

Theorem 1.1: Let $u_{0}, v_{0} \in \mathrm{H}^{2}\left(\mathfrak{R}_{+}\right)$be such that

$$
u_{0}(0)=v_{0}(0), \frac{\partial u_{0}}{\partial r}(0)=-\frac{\partial v_{0}}{\partial r}(0) \text { and } r u_{0}, r v_{0} \in L_{-}^{2}\left(\mathfrak{R}_{+}\right) .
$$

Then, there exists $(u, v)$ such that $r u, r v \in \mathrm{L}^{\infty}\left(\Re, \mathrm{L}^{2}\left(\mathfrak{R}_{+}\right)\right), r^{2} u, r^{2} v \in$ $\mathrm{C}\left([-T, T] ; \mathrm{L}^{2}(] 0, R[)\right) \cap \mathrm{L}^{2}\left(-T, T ; \quad \mathrm{H}_{0,}^{1}, \quad(] 0 . \mathrm{R}[)\right), \frac{\partial u}{\partial v}, \frac{\partial u}{\partial t} \in \mathrm{L}^{2}(-T ; T ;$ $\left.\mathrm{L}^{2}(] 0, R[)\right)$, for each $R>0$ and $T>0, u(r, 0)=u_{0}(r), v(r, 0)=v_{0}(r), r \in \mathfrak{R}_{+}$, and $(u, v)$ verifies $(1.1)$ in $\left(\mathcal{O}^{\prime}\left(\mathfrak{R}_{+} \times \mathfrak{R}\right)\right)^{2}$.

Our previous papers [2] and [3] are concerned with nonlocal nonlinear complex perturbations (nonlinear Dirac system) of the principal part of the system (1.1). In this paper we deal with a local nonlinear perturbation which is, as far as we know, the only one that can be analysed by this method. 


\section{ESTIMATES FOR THE REGULARISED PROBLEM}

As in [2] and [3], we consider the skew-adjoint operator in $\left(\mathrm{L}^{2}\left(\mathfrak{R}_{+}\right)\right)^{2}$ defined by

$$
\begin{gathered}
D(A)=\left\{\left(\begin{array}{l}
u \\
v
\end{array}\right) \in\left(\mathrm{H}^{i}\left(\mathfrak{R}_{+}\right)\right)^{2} \mid u-v \in \mathrm{H}_{0}^{1}\left(\mathfrak{R}_{+}\right)\right\} . \\
A\left(\begin{array}{l}
u \prime \\
v
\end{array}\right)=\left(\begin{array}{c}
-\frac{\partial u}{\partial r} \\
\frac{\partial v}{\partial r}
\end{array}\right) .
\end{gathered}
$$

We put $S(t)=e^{x+t}, t \in \mathfrak{K}$, and $S(t)$ is defined by

$$
S(t)\left(\begin{array}{l}
u \\
v
\end{array}\right)=\left(\begin{array}{l}
u(r-t) \\
v(r+i)
\end{array}\right) \text { with }\left\{\begin{array}{l}
u(-r)=v(r) \\
v(-r)=u(r)
\end{array}, r>0\right.
$$

We have, in $D(A)$ (with $\left.L^{\mathrm{r}}=\left(\mathrm{L}^{\mathrm{P}}\left(\mathfrak{R}_{+}\right)\right)^{2}, \mathrm{H}^{\mathrm{m}}=\left(\mathrm{H}^{\mathrm{m}}\left(\mathfrak{R}_{+}\right)\right)^{2}\right)$,

$$
\left\|S(t)\left(\begin{array}{l}
l t \\
b
\end{array}\right)\right\|_{1^{*}}=\left\|\left(\begin{array}{l}
u \\
v
\end{array}\right)\right\|_{1^{*}},\left\|S(t)\left(\begin{array}{l}
u \\
v
\end{array}\right)\right\|_{\mathrm{H}^{1}}=\left\|\left(\begin{array}{l}
u \\
v
\end{array}\right)\right\|_{\mathbb{L}^{1}, t \in \mathcal{R}} .
$$

Now, let be $F: D(A) \rightarrow D(A), D(A)$ with the $\mathrm{H}^{\prime}$ norm, defined by

$$
F\left(\begin{array}{l}
u \\
v
\end{array}\right)=-\frac{1}{\overline{r+\delta}}\left[\begin{array}{l}
1-1 \\
1-1
\end{array}\right]\left(\begin{array}{l}
u \\
v
\end{array}\right)-k\left(u^{2}+v^{2}\right)\left(\begin{array}{l}
u \\
v
\end{array}\right)
$$

This map is locally Lipschitz continuous. Hence, for $\left(\begin{array}{l}u_{0} \\ v_{0}\end{array}\right) \in D(A)$ there exists $T>0$ and an unique $\left(\begin{array}{l}u \\ v\end{array}\right) \in \mathrm{C}([-T, T] ; D(A)) \cap \mathrm{C}^{\prime}\left([-T, T] ; \mathrm{L}^{2}\right)$ such that

$$
\left(\begin{array}{l}
u \\
v
\end{array}\right)=S(t)\left(\begin{array}{l}
u_{0} \\
v_{0}
\end{array}\right)+\int_{0}^{t} S(t-\tau) F\left[\left(\begin{array}{l}
u \\
v
\end{array}\right)(\tau)\right] \mathrm{d} \tau
$$

We have $\left\|F\left(\begin{array}{l}u \\ v\end{array}\right)\right\|_{H^{1}} \leq c(\delta)\left(1+\left\|\left(\begin{array}{l}u \\ v\end{array}\right)\right\|_{L^{\infty}}^{2}\right)\left\|\left(\begin{array}{l}u \\ v\end{array}\right)\right\|_{H_{1}}$, and, for $t \in[-T, T]$,

$$
\left\{\begin{array}{l}
\frac{\partial}{\partial t} u^{2}+\frac{\partial}{\partial r}-u^{2}+\frac{2}{r+\delta}(u-v) u+2 k\left(u^{2}+v^{2}\right) u^{2}=0 \\
-\frac{\partial}{\partial t} v^{2}-\frac{\partial}{\partial r} v^{2}+\frac{2}{r+\delta}(u-v) v+2 k\left(u^{2}+v^{2}\right) v^{2}=0
\end{array}\right.
$$


Hence, since $\left(\begin{array}{l}u^{2} \\ v^{2}\end{array}\right) \in \mathrm{C}([-T, T] ; D(A)) \cap \mathrm{C}^{\prime}\left([-T, T] ; \mathrm{L}^{2}\right)$ and $k \geq 0$,

$$
\begin{gathered}
\left(\begin{array}{l}
u^{2} \\
v^{2}
\end{array}\right)(t) \leq S(t)\left(\begin{array}{l}
u^{2} v^{0} \\
2_{0}
\end{array}\right)-\int_{0}^{t} S(t-\tau)\left[\frac{2}{r+\delta}\left(\begin{array}{c}
(u-v) u \\
(u-v) v
\end{array}\right)(\tau)\right] \mathrm{d} \tau, t \in[0, T], \\
\left\|\left(\begin{array}{c}
u^{2} \\
v^{2}
\end{array}\right)(t)\right\|_{L^{\infty}} \leq c\left\|\left(\begin{array}{l}
u^{2} v^{2} \\
v_{0}
\end{array}\right)\right\|_{L^{\infty}}+\frac{c}{\delta} \int_{0}^{t}\left\|\left(\begin{array}{c}
t^{2} \\
v^{2}
\end{array}\right)(\tau)\right\|_{L^{\infty}} \mathrm{d} \tau, t \in[0, T], \\
\left\|\left(\begin{array}{l}
u^{2} \\
v^{2}
\end{array}\right)(t)\right\|_{L^{\infty}} \leq c e^{(c / \delta) t}, t \in[0, T]
\end{gathered}
$$

From (2.1) and (2.2) we easily obtain an estimate for $\left\|\left(\begin{array}{l}u \\ b\end{array}\right)(r)\right\|_{\mathrm{H}^{1}}$ for $t \in[0, T]$ (and also for $t \in[-T, 0])$ and we conclude that $\left(\begin{array}{l}u \\ v\end{array}\right)$ is a global solution, that is

$\left(\begin{array}{l}u \\ v\end{array}\right) \in \mathrm{C}(\mathfrak{R} ; D(A)) \cap \mathrm{C}^{1}\left(\mathfrak{R} ; \mathrm{L}^{2}\right)$ and verifies $(2.1)$ for $t \in \mathfrak{R}$.

Furthermore, since $F: D(A) \rightarrow D(A)$ is locally Lipschitz continuous and $D(A)$ is a Hilbert space (for the $\mathrm{H}^{\dagger}$ norm), we get (cf. [1]) $\left(\begin{array}{l}u \\ v\end{array}\right) \in \mathrm{C}^{1}(\mathbb{R} ; D(A))$ if

$$
\left(\begin{array}{l}
u_{0} \\
v_{0}
\end{array}\right) \in D\left(A^{2}\right)=\left\{\left(\begin{array}{l}
u \\
v
\end{array}\right) \in \mathbf{H}^{2} \mid u-v \in \mathbf{H}_{0}^{1}\left(\boldsymbol{R}_{+}\right), \frac{\partial u}{\partial r}+\frac{\partial v}{\partial r} \in \mathbf{H}_{0}^{1}\left(\mathfrak{R}_{+}\right)\right\} .
$$

Hence, by (1.2), we obtain, in this case $\left(\begin{array}{l}u \\ v\end{array}\right) \in \mathrm{C}\left(\mathfrak{R} ; D\left(A^{2}\right)\right)$.

We need suplementary estimates for $\left(\begin{array}{l}u \\ v\end{array}\right)$. For this purpose we assume that $\left(\begin{array}{c}r u_{0} \\ r v_{0}\end{array}\right) \in \mathrm{L}^{2}$, that is

$$
\left(\begin{array}{l}
u_{0} \\
v_{0}
\end{array}\right) \in\left(\mathrm{L}_{r}^{2}\left(\mathfrak{R}_{+}\right)\right)^{2} \text {, where } \mathrm{L}_{r}^{2}\left(\mathfrak{R}_{+}\right)=\mathrm{L}^{2}\left(\mathfrak{R}_{+}, r^{2} d r\right)
$$

We easily deduce from (1.2), since $k \geq 0$,

$$
\left\{\begin{array}{l}
\frac{\partial}{\partial t}\left(u^{2}+v^{2}\right)+\frac{\partial}{\partial r}\left(u^{2}-v^{2}\right)+\frac{2}{r+\delta}\left(u^{2}-v^{2}\right) \leq 0, \\
\frac{\partial}{\partial t}\left((r+\delta)^{2}\left(u^{2}+v^{2}\right)\right)+\frac{\partial}{\partial r}\left((r+\delta)^{2}\left(u^{2}-v^{2}\right)\right) \leq 0 .
\end{array} .\right.
$$

By the integral of energy method we get, for $R>0,0<t<R$,

$$
\int_{0}^{-r+R}(r+\delta)^{2}\left(u^{2}+v^{2}\right)(r, t) d r \leq \int_{0}^{R}(r+\delta)^{2}\left(u_{0}^{2}+v_{0}^{2}\right) d r \leq \int_{\pi_{r}}(r+\delta)^{2}\left(u_{0}^{2}+v_{0}^{2}\right) d r .
$$

Hence $(r+\delta)(u, v) \in L^{\infty}\left(\Re, L^{2}\right)$ and 


$$
\left\|(r+\delta)\left(\begin{array}{l}
u \\
v
\end{array}\right)\right\|_{\mathrm{L}^{\infty}\left(\mathcal{R} ; \mathrm{L}^{2}\right)} \leq c_{1}\left(\left\|\left(\begin{array}{c}
u_{0} \\
v_{0}
\end{array}\right)\right\|_{\mathrm{1}_{-r}^{2}}+\left\|\left(\begin{array}{c}
u_{0} \\
v_{0}
\end{array}\right)\right\|_{\left.\mathrm{L}^{2}\right)}\right.
$$

where $c_{1}$ does not depend on $\delta$.

Now, let us consider the linear system in D:

$$
\left\{\begin{array}{l}
\frac{\partial u}{\partial t}+\frac{\partial u}{\partial r}+\frac{1}{r}(u-v)=0 \\
\frac{\partial v}{\partial t}-\frac{\partial v}{\partial r}+\frac{1}{r}(u-v)=0
\end{array}\right.
$$

L. Tartar has pointed out to us that if we put

$$
\left\{\begin{array}{l}
w=r u_{r}+\frac{3}{2} u-\frac{1}{2} v \\
w_{1}=r v_{r}+\frac{3}{2} v-\frac{1}{2} u
\end{array}, \text { where } u_{r}=\frac{\partial u}{\partial r},\right.
$$

we obtain (formally): $\left\{\begin{array}{l}\frac{\partial w^{\prime}}{\partial t}+\frac{\partial w}{\partial r}=0 \\ \frac{\partial w_{1}}{\partial t}-\frac{\partial w_{1}}{\partial r}=0\end{array}\right.$.

Let us assume $\left(u_{0}, v_{0}\right) \in D\left(A^{2}\right) \cap \mathrm{L}_{r}^{2}$ and let $(u, v) \in \mathrm{C}\left(\mathfrak{R} ; D\left(A^{2}\right)\right) \cap \mathrm{C}^{1}(\mathfrak{R}$; $D(A)) \cap L^{\infty}\left(\Re ; L_{r}^{2}\right)$ be the solution of (1.2) for a fixed $\left.\delta \in\right] 0,1[$ and with initial data $\left(u_{0}, v_{0}\right)$ (and boundary condition (1.3)). Let

$$
\left\{\begin{array}{l}
w=(r+\delta) u_{r}+\frac{3}{2} u-\frac{1}{2} v \\
w_{1}=(r+\delta) v_{r}+\frac{3}{2} v-\frac{1}{2} u
\end{array}\right.
$$


We deduce, from (1.2), with $\theta=k\left(u^{2}+v^{2}\right)$,

$$
\left\{\begin{array}{l}
\frac{\partial w}{\partial t}+\frac{\partial w^{v}}{\partial r}=-\theta w_{-}(r+\delta) \theta_{r} u \\
\frac{\partial w_{1}}{\partial t}-\frac{\partial w_{1}}{\partial r}=-\theta w_{1}-(r+\delta) \theta_{r} w
\end{array}\right.
$$

and $\left.\left(w_{1}, w_{1}\right)=\mathrm{C}\left(\Re:\left(\mathrm{H}^{1}(] 0, R[)\right)^{2}\right) \cap \mathrm{C}^{\prime}\left(\mathfrak{R}:\left(\mathrm{L}^{2}(]\right), R[)\right)^{2}\right)$. for each $R \in] 0,+\infty[$.

Furthermore, we have

$$
\left\{\begin{array}{l}
\frac{\partial}{\partial r}[(r+\delta)(u+v)]=w+w_{1} \\
(u-v)+\frac{\partial}{\partial r}[(r+\delta)(u-v)]=w-w_{1}
\end{array}\right.
$$

and

$$
\left\{\begin{array}{l}
\frac{\partial}{\partial r}\left[(r+\delta)^{2} u\right]=(r+\delta) w+\frac{1}{2}(r+\delta)(u+v) \\
\frac{\partial}{\partial r}\left[(r+\delta)^{2} v\right]=(r+\delta) w_{1}+\frac{1}{2}(r+\delta)(u+\cdots)
\end{array}\right.
$$

By applying the Gagliardo-Nitenberg inequalities and the Sobolev theorem to $(r+\delta)(u+v) \in \mathrm{H}^{\prime}(] 0, R[)$ and $\left.(r+\delta)^{2}(u-v)^{2} \in W^{1.1}\right] 0, R[)$, respectively, we can deduce from (2.6). (1.3) and (2.3) (cl.[3]. \$3):

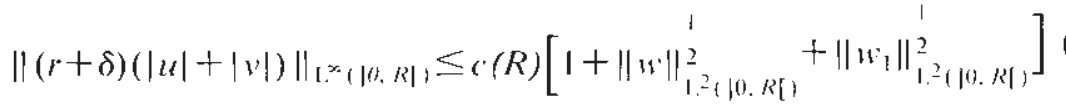

where $r(R)$ does not depend on $\delta$.

From (2.5) we deduce

$$
\begin{aligned}
& \frac{\partial}{\partial t}\left(\left.\left|w^{2}+\right| w_{1}\right|^{2}\right)+\frac{\partial}{\partial r}\left(\left.\left|w^{2}-\right| w_{1}\right|^{2}\right)= \\
& -2 \theta\left(w^{2}+w^{2}\right)-2(r+\delta) \theta_{r}\left(w+w_{1}\right)
\end{aligned}
$$


and $\theta_{r}=h^{\prime}\left(u^{2}+v^{2}\right)+2 k\left(u u_{r}+v v_{s}\right)=k^{\prime}\left(u^{2}+v^{2}\right)+2 \frac{k}{r+\delta}\left[u w^{\prime}+v w_{1}-\right.$ $\left.-\frac{3}{2}\left(w^{2}+v^{2}\right)+w\right]$.

Hence,

$-2(r+\delta) \theta_{r}\left(u w+v w_{1}\right)=-2(r+\delta)\left(\frac{k^{\prime}}{r^{2}}\right) r^{2}\left(u^{2}+v^{2}\right)\left(u w^{2}+w_{1}\right)-$ $-4 k\left(u w+w_{1}\right)^{2}-4 \frac{k}{r^{3}} \cdot r^{3}\left[-\frac{3}{2}\left(u^{2}+v^{2}\right)+u v\right]\left(u w+v w_{1}\right)$

Then we deduce. from (2.9) and from the properties of $k$.

$\frac{\partial}{\partial t}\left(|\pi|^{2}+\left|w_{1}\right|^{2}\right)+\frac{\partial}{\partial r}\left(\left|w^{2}\right|^{2}-\left|w_{1}\right|^{2}\right) \leq c(r+\delta)^{3}\left(|u|^{3}+|v|^{3}\right)\left(\left|w^{2}\right|+\left|w_{1}\right|\right)$

where $c$ does not depend on $\delta$.

Furthermore, we have, by (2.3) and (2.8).

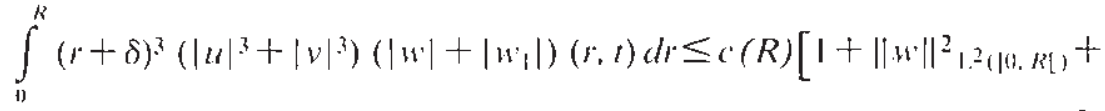

$$
\begin{aligned}
& +\left\|w_{1}\right\|^{2}, ?[(1), N[n]
\end{aligned}
$$

where $c(R)$ does not depend on $\delta$.

Now, if we take $T>0$ we easily obtain, by applying the integral of energy method to $(2.10)$, and since $\left|w_{1}\right|^{2}(0, \tau)-|w|^{2}(0, \tau)=2 \delta\left(\frac{\partial}{\partial t}-|u|^{2}\right)(0, \tau)$ (cf. [2]. \$3).

$$
\int_{0}^{-t+2 t}\left(\left|w_{1}\right|^{2}+\left|w_{1}\right|^{2}\right)(r, t) d r \leq \ell(T) \text {, for all } t \in[0, T]
$$

Hence, if $\Omega=\{(r, t) \mid r \in[0,-t+2 T[, t \in] 0, T[\}$, we deduce

$$
\int_{12,}\left(|w|^{2}+\left|w_{1}\right|^{2}\right)(r, t) d r d t \leq r(T)
$$


where $c(T)$ does not depend on $\delta$. A similar estimate holds for $T<0$. Now, for every $T>0$ and $R>0$, we deduce from (2.12), (2.7) and (2.3),

$$
\left\{\begin{array}{l}
\left\|(r+\delta)^{2} u\right\|_{\mathrm{L}^{2}\left(-T . T: \|^{\prime}(j 0 . R[n)\right.} \leq c(R, T) \\
\left\|(r+\delta)^{2} v\right\|_{\mathrm{L}^{2}\left(-T . T: \|^{\prime}(\mid), R[1)\right.} \leq c(R, T)
\end{array}\right.
$$

Hence, by (2.3), (2.13) and (1.2), we obtain

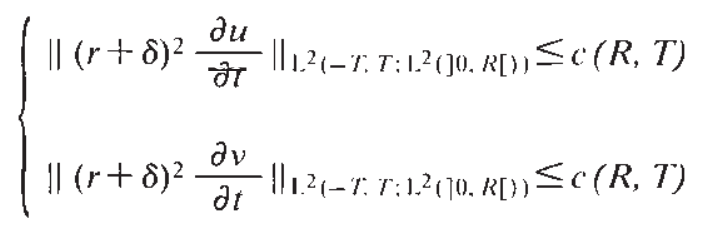

where $c(R, T)$ does not depend on $\delta$.

Now, let

$$
W(R, T)=\left\{u \in \mathrm{L}^{2}\left(-T, T: \mathrm{H}_{0}^{\dagger},(] 0, R[)\right) \mid \frac{\partial u}{\partial t} \in \mathrm{L}^{2}\left(-T, T ; \mathrm{L}^{2}(] 0, R[)\right)\right\}
$$

with its natural norm, where $\mathrm{H}_{0,}^{1}(] 0, R[)=\left\{u \in \mathrm{H}^{\prime}(] 0, R[) \mid u(0)=0\right\}$. We have (cf. [4]).

$$
W(R, T) \subset \mathrm{C}\left([-T, T] ; \mathrm{L}^{2}(] 0, R[)\right)
$$

and, by Aubin's compactness theorem (cf. [4])

$W(R, T) \subseteq \mathrm{L}^{2}\left(-T ; T ; \mathrm{L}^{2}(] 0, R[)\right)$, with compact injection

Furthermore the map $u \rightarrow u(0)$ from $W(R, T)$ into $\mathrm{L}^{2}(] 0, R[)$ is continuous by $(2.15)$.

Let, for each $\delta \in] 0,1\left[\right.$, be $\left(u_{\delta}, v_{\delta}\right)$ the corresponding solution of (1.2) for the initial data $\left(u_{0}, v_{0}\right)$ (and boundary condition (1.3)). We have, by (2.3). $(2.13)$ and (2.14),

$$
\left\{\begin{array}{l}
\left\|r^{2} u_{\delta}\right\|_{w(R, T)} \leq c(R, T) \\
\left\|r^{2} v_{\delta}\right\|_{w^{\prime}(R, T)} \leq c(R, T)
\end{array}\right.
$$

where $c(R, T)$ does not depend on $\delta$. 


\section{EXISTENCE OF A WEAK SOLUTION}

Let us assume $\left(u_{0}, v_{0}\right) \in D\left(A^{2}\right) \cap \mathrm{L}_{r}^{2}$. With the same notation of the last part of $\S 2$ for the couple $\left(u_{\delta}, v_{\delta}\right)$, solution of the regularised problem, there exists, by (2.3), a sequence $\delta \rightarrow 0$ and $(u, v) \in \mathrm{L}^{\infty}\left(\mathcal{R}, \mathrm{L}_{r}^{2}\right)$ such that

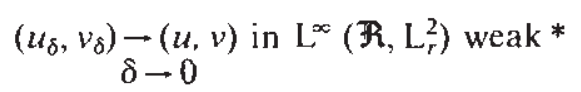

and

$$
\|(u, v)\|_{L_{\infty}^{\infty}\left(\mathcal{A} . L_{r}^{2}\right)} \leq c\left(\left\|\left(u_{0}, v_{0}\right)\right\|_{\mathrm{L}_{-}^{2}}+\left\|\left(u_{0}, v_{0}\right)\right\|_{\mathrm{L}_{-2}}\right) .
$$

By (2.17), (2.16) and (2.15) there exists, for each $(R . T)$, a sub-sequence $\left\langle u_{\delta}, v_{\delta}\right), \delta \rightarrow 0$, such that

$\left(r^{2} u_{\delta}, \quad r^{2} v_{\delta}\right) \rightarrow\left(r^{2} u, r^{2} v\right)$ weakly in $(W(R, T))^{2}, \quad$ strongly in $\left(\mathrm{L}^{2}\left(-T, T ; L^{2}(] 0, R[)\right)\right)^{2}$ and a.e. in $] 0, R[\times]-T, T\left[\right.$, and $(u, v)(0)=\left(u_{0}, v_{0}\right)$ a.e. in $\mathfrak{R}_{+}$. Furthermore, for each $T>0$, and by a diagonalisation method, we can assume (by $(2.16)$ and $(2.17))$ that

$$
\left.\left(u_{\delta}, v_{\delta}\right) \underset{\delta \rightarrow 0}{\rightarrow}(u, v) \text { a.e. in } \mathfrak{f}_{+} \times\right]-T, T[.
$$

In particular, for fixed $T>0$, we have, for each $R>0$,

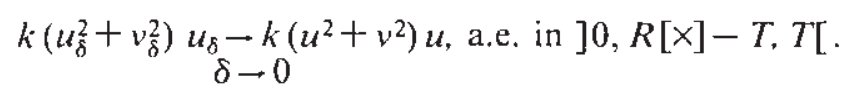

Otherwise, by (2.12) and (2.8), we have

$\| k\left(\left(u_{\delta}^{2}+v_{\delta}^{2}\right) u_{\delta}\left\|_{L_{2}^{2}(\mid \theta, R[\times]-\tau, \pi)} \leq c\right\| r^{3}\left(\left(u_{\delta}^{2}+u_{\delta}^{2}\right) u_{\delta} \|_{\left.L^{2}(] 0, R[\times]-T, \pi\right)} \leq c(R, T)\right.\right.$, where $c(R, T)$ does not depend on $\delta$.

Hence, by lemma 1.3 in chap. 1 of [4],

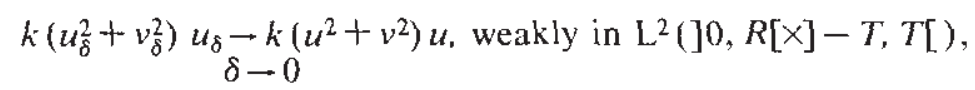

and similar conclusion for $k\left(u_{\delta}^{2}+v_{\delta}^{2}\right) v_{\delta}$.

Now, take $\phi \in \mathscr{Z}(\mathrm{D}(R, T)), \mathrm{D}(R, T)=\{(r, t) \mid r \in] 0, R[, t \in]-T, T[\}$. we have, by (1.2), 


$$
\int_{-r}^{r} \int_{i}^{k}\left[\frac{\partial u_{\delta}}{\partial r} \phi+\frac{\partial u_{i}}{\partial r} \phi+\frac{u_{\delta}-v_{\delta}}{\mu+\delta} \phi+k\left(u_{\delta}^{2}+v_{\delta}^{2}\right) u_{\delta} \phi\right] d r d t=0
$$

By the previous considerations we can pass to the limit, when $\delta \rightarrow 0$ (subsequence) and we obtaim. since $\mathrm{R}$ and $\mathrm{T}$ are arbitrary,

$$
\frac{\partial u}{\partial t}+\frac{\partial u}{\partial r}+\frac{u-v}{r}+k\left(u^{2}+v^{2}\right) u=0 \text { in } \gamma^{\prime}(\mathrm{D}) \text {. }
$$

The same technique applies to the second equation, and so theorem 1.1 is proved.

\section{References}

[1] T. CAzENAVE and F. Wulssigk. The Cauchl problem for the nonlined Sidrödinger conarion in $/{ }^{\prime}$. Manuseripta Math., 61 (1988), 477.494.

[2] J. P. DIss and M. Figiulars. On the cersteme of weak solutions for a nomlineat time dependem Dirac equation. Proc. Royal Soc. Edinburgh, 113 A (1989), 149158

[3] J. P. DIAs and M. FigulaIRA. Solutions fables do probleme de Candy pour

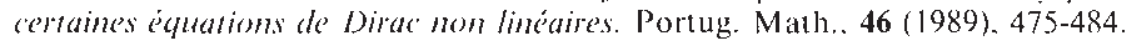

[4] I. L. I.Ions. Quelques méhodes de reswlution des problemes and limites non linécoires. Paris, Dunod. 1969.

\footnotetext{
$(\mathrm{NAN}$

2 Aid, prod (jimm Jimls.

low9 Lisbona Code:

Porluyial
} 